Abstract 0-031 Table 2 MVP and RI in patients (pre and poststenting)

\begin{tabular}{lcll}
\hline & Pre stenting $\mathrm{n}=11$ & Post stenting $\mathrm{n}=11$ & P value \\
\hline Mean venous sinus pressure & (MVP) $\mathbf{m m H g}$ & & \\
Superior sagittal sinus & $41.9 \pm 14.6$ & $22.5 \pm 8.4$ & $<0.001$ \\
Transverse sinus & $39.4 \pm 15.1$ & $19.5 \pm 8.2$ & $<0.001$ \\
Sigmoid sinus & $16.3 \pm 6.2$ & $17.5 \pm 6.7$ & 0.23 \\
Pressure difference across the & $22.8 \pm 13.3$ & $1.8 \pm 1.1$ & $<0.001$ \\
stenosis & & & \\
Resistivity index (RI) & & & \\
Superior sagittal sinus & $0.22 \pm 0.13$ & $0.17 \pm 0.11$ & $<0.01$ \\
Transverse sinus & $0.23 \pm 0.13$ & $0.17 \pm 0.08$ & 0.03 \\
Sigmoid sinus & $0.16 \pm 0.05$ & $0.16 \pm 0.11$ & 0.84 \\
\hline
\end{tabular}

All values are presented as (mean \pm SD). Boldface type indicates statistical significance

Abstract 0-031 Table 3 RI changes across stenosis (pre and post-stenting)

\begin{tabular}{llll}
\hline & $\begin{array}{l}\text { Resistive index } \\
\text { Proximal to stenosis }\end{array}$ & Resistive index distal to stenosis & P value \\
\hline Pre-stenting & $0.24 \pm 0.12$ & $0.15 \pm 0.06$ & 0.03 \\
Post-stenting & $0.17 \pm 0.09$ & $0.15 \pm 0.1$ & 0.66 \\
\hline
\end{tabular}

All values are presented as (mean $\pm \mathrm{SD}$ ). Boldface type indicates statistical significance

Methods Retrospective evaluation of 13 consecutive $\mathrm{IIH}$ patients who underwent venous sinus stenting at our institution between 2013-2018 was reviewed.

Patients' demographics, clinical presentation, cerebral mean venous sinus pressure (MVP) and RI both pre and post-stenting were recorded. The baseline RI was also compared to a control group.

Results Among 13 patients of IIH, 11 had unilateral VSS in dominant sinus, while 2 had bilateral VSS. RI was significantly higher in IIH patients compared to the control group in the superior sagittal (SSS) and transverse sinuses (TS) (0.21 vs $0.11, \mathrm{p}=0.01$ and 0.22 vs $0.13, \mathrm{p}=0.03$, respectively). The MVP (in $\mathrm{mmHg}$ ) decreased significantly after venous sinus stenting in the SSS $(41.9$ to $22.5, \mathrm{p}<0.001)$ and TS (39.4 to $19.5, \mathrm{p}<0.001)$ which was also associated with a significant reduction of the RI $(0.22$ vs. $0.17, \mathrm{p}<0.01$ in SSS and 0.23 vs. $0.17, p=0.03$ in TS) post-stenting.
Conclusion RI calculated using QMRV can serve as a noninvasive tool to aid in the diagnosis of hemodynamically significant VSS. The study had a small sample size, and larger multicenter studies would be required to validate the results further.

Disclosures M. Mohammaden: None. M. Rizwan Husain: None. D. Brunozzi: None. G. Atwal: None. F. Charbel: None. A. Alaraj: 1; C; NIH. 2; C; Cerenovus.

\section{0-032 MICROCATHETER DELIVERY OF NEUROTHERAPEUTICS: COMPATIBILITY WITH MESENCHYMAL STEM CELLS}

${ }^{1} \mathrm{~V}$ Srinivasan*, ${ }^{1} \mathrm{~J}$ Gumin, ${ }^{1} \mathrm{~K}$ Camstra, ${ }^{2} \mathrm{~S}$ Chen, ${ }^{1} \mathrm{~J}$ Johnson, ${ }^{1} \mathrm{~F}$ Lang, ${ }^{1} \mathrm{P}$ Kan. ${ }^{1}$ Neurosurgery, Baylor College of Medicine, Houston, TX; ${ }^{2}$ Radiology, Baylor College of Medicine, Houston, TX

\subsection{6/neurintsurg-2019-SNIS.32}

Background Mesenchymal stem cells (MSCs) are being studied for the treatment of several neurological disorders. The use of MSCs as delivery modality for targeted viral therapeutic agents for the treatment of intracranial pathologies has not been explored. Delta-24-RGD, a tumor-selective oncolytic adenovirus designed to target malignant glioma cells, has been shown effective in animal models. However, the most efficient viral tissue delivery modality is unclear. Bone marrow human MSCs (BM-hMSC) have been shown to have homing capability toward glioma xenografts and the feasibility of oncolytic virus particles loaded in MSCs being delivered to the tumor vascular bed via selective intra-arterial (IA) infusion is being investigated. To evaluate the feasibility of endovascular IA delivery, catheter compatibility with MSCs was tested in vitro. Methods BM-hMSCs were cultured, transfected with Delta-24, and re-suspended in 1\% Human Serum Albumin. hMSCDelta-24 solution was then injected via three microcatheters of different inner diameters (Marathon, Echelon-14, and Marksman). Cell count and viability after injection through the microcatheters based on injection velocity and catheter configuration were assessed. Transwell assay was performed with the injected cells to test the Delta-24 activity against U87 glioma cells. hMSC-Delta-24 compatibility was also tested with commonly used medications in neuroendovascular therapy (Omnipaque, verapamil, and heparin).

Results BM-hMSC cell count prior to infusion was $0.123 \times$ $10^{6}$ cells $/ \mathrm{mL}, 98.7 \%$ viability. There was no significant difference in cell count after infused through any of the catheters

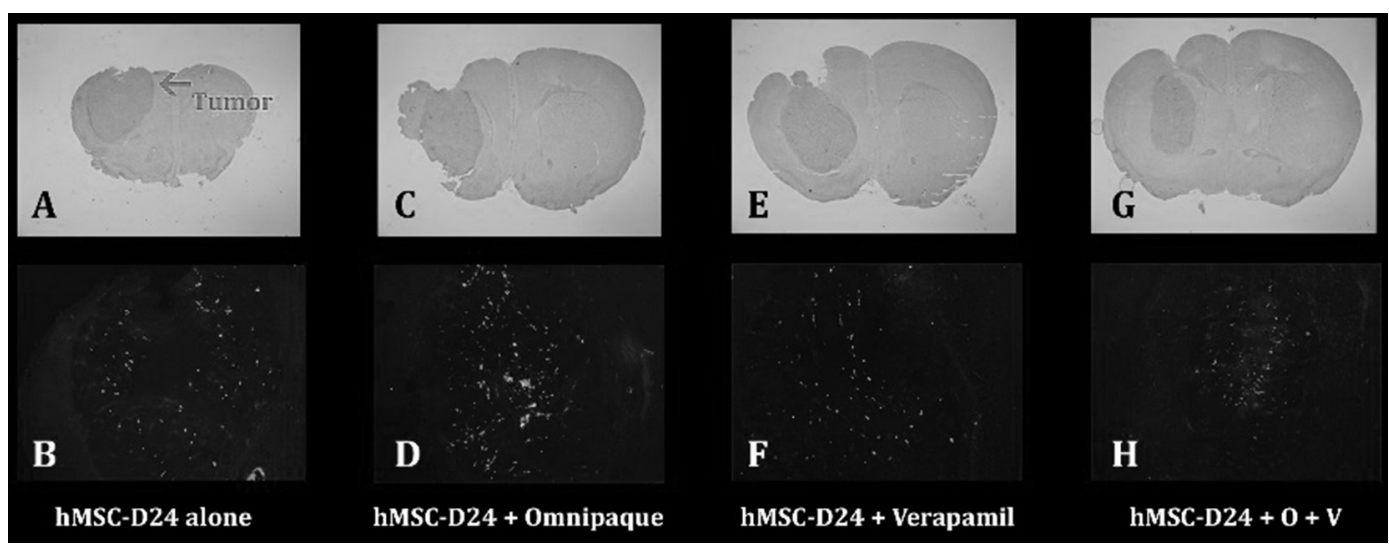

Abstract 0-032 Figure 1 H\&E slides (top row, A, C, E, G) and corresponding GFP-stained sections (bottom row, B, D, F, H) of nude mouse cerebri. The magnified GFP sections show diffuse localization of the GFP-labeled Delta-24-hMSCs throughout the tumor following IA injection 
under standard conditions, with a mean concentration of $0.126 \times 10^{6}$ cells $/ \mathrm{mL}$ and $97.9 \%( \pm 1.7 \%)$ viability. Injection velocity ranged from 1.01 to $73.17 \mathrm{cc} / \mathrm{min}$, with no significant difference in cell count or viability. The same result was seen in either tortuous or straight microcatheter configurations. Cell count and viability did not change significantly when the hMSC in solution was stored for up to 5 hours on ice or mixed with Omnipaque, verapamil, and heparin. Notably, antiglioma activity was maintained after microcatheter infusion.

Conclusions BM-hMSCs are compatible with a wide variety of commonly used neuroendovascular microcatheters and medications. Stem cell viability and viral agent activity do not appear to be affected by catheter configuration or injection velocity. Commercially available microcatheters can be used to deliver IA stem cell neurotherapeutics.

Disclosures V. Srinivasan: None. J. Gumin: None. K. Camstra: None. S. Chen: None. J. Johnson: None. F. Lang: None. P. Kan: 2; C; Stryker, Medtronic, Cerenovus.

\section{0-033 POST-EMBOLIZATION CONTRASTED MRI ENHANCEMENT OF MENINGIOMAS IS A GREATER PREDICTOR OF INTRAOPERATIVE BLOOD LOSS THAN ANGIOGRAPHY}

J Catapano*, C Pryzbylowski, A See, A Whiting, M Labib, N Rubel, V Fredrickson, A Ducruet, F Albuquerque, N Sanai. Neurosurgery, Barrow Neurological Institute, Phoenix, $A Z$

\subsection{6/neurintsurg-2019-SNIS.33}

Objective Pre-operative embolization of meningiomas remains controversial. Prior studies have shown that the degree of devascularization on angiography is not significantly correlated with intraoperative blood loss. This study examines pre and post-embolization MRI enhancement as an improved metric for assessing the degree of embolization.

Methods We retrospectively analyzed patients who underwent preoperative embolization for intracranial meningiomas at the Barrow Neurological Institute from 2007 to 2017. Two cohorts were analyzed based on the degree of devascularization observed $(>/=50 \%$ vs. $<50 \%)$.

Results 84 meningioma patients underwent preoperative embolization. 35 (42\%) had post-embolization MR imaging prior to surgical resection. The mean lesion diameter was 4.9 $\mathrm{cm}( \pm 1.3)$ and, intraoperatively, the mean blood loss was $576 \mathrm{ml}( \pm 341)$. Based on MR imaging, angiography overestimated devascularization in 22 patients (63\%). 17 (49\%) patients were found to have a $>/=50 \%$ decrease enhancement on contrasted post-embolization MRI which was associated with lower mean intraoperative blood loss [444 $\mathrm{ml}$ vs. $700 \mathrm{ml}$ in 17 patients with $<50 \%$ devascularization $(\mathrm{p}=0.025)]$. On angiography, 22(63\%) patients demonstrated $>/=50 \%$ devascularization during embolization. These patients did not statistically differ in intraoperative blood loss as compared to those with $<50 \%$ devascularization on angiography. On univariate analysis, patients with $<50 \%$ decrease in enhancement on contrasted post-embolization MRI had 9 times greater odds of having at least $500 \mathrm{cc}$ of intraoperative blood loss during resection (CI 1.6-54, $\mathrm{p}=0.012$ ). Additionally, on multivariate stepwise logistic regression analysis $<50 \%$ decrease enhancement on contrasted post-embolization MRI was found to be a predictor of at least $500 \mathrm{cc}$ of blood loss (OR 9.4, p =0.012), but $<50 \%$ devascularization on angiography was not.
Conclusion Post-embolization contrasted MRI is a better predictor of intraoperative blood loss during meningioma resection than post-embolization angiography, which overestimates the degree of devascularization.

Disclosures J. Catapano: None. C. Pryzbylowski: None. A. See: None. A. Whiting: None. M. Labib: None. N. Rubel: None. V. Fredrickson: None. A. Ducruet: None. F. Albuquerque: None. N. Sanai: None.

\section{0-034 THROMBECTOMY FOR ACUTE ISCHEMIC STROKE IN NONAGENARIANS COMPARED TO OCTOGENARIANS}

${ }^{1}$ E Sussman*, ${ }^{2} B$ Martin, ${ }^{3} \mathrm{M}$ Mlynash, ${ }^{2} \mathrm{M}$ Marks, ${ }^{2} \mathrm{D}$ Marcellus, ${ }^{3} \mathrm{G}$ Albers, ${ }^{3} \mathrm{M}$ Lansberg, ${ }^{1} \mathrm{R}$ Dodd, ${ }^{2} \mathrm{H}$ Do, ${ }^{2} \mathrm{~J}$ Heit. ${ }^{1}$ Neurosurgery, Stanford University, Stanford, $C A$; ${ }^{2}$ Radiology, Stanford University, Stanford, $C A ;{ }^{3}$ Neurology, Stanford University, Stanford, $C A$

\subsection{6/neurintsurg-2019-SNIS.34}

Introduction Recent landmark randomized clinical trials have demonstrated that endovascular thrombectomy (EVT) leads to improved outcomes in patients with acute ischemic stroke (AIS) due to large vessel occlusion (LVO). Although elderly patients were excluded from several of these initial trials, the available data suggests a benefit of EVT in octogenarian patients with AIS due to LVO. However, the efficacy of EVT in the nonagenarian patient population remains uncertain.

Methods We performed a retrospective cohort study of a prospectively-maintained stroke database at a single comprehensive stroke center. Inclusion criteria were: age 80-99 years, LVO, core infarct $<70 \mathrm{~mL}$ on perfusion imaging, and presence of a salvageable penumbra. Patients were stratified based on age into octogenarian (age 80-89) and nonagenarian (age 90-99) cohorts. Primary outcome was ordinal score on the modified Rankin Scale (mRS) at 90 days. Secondary outcomes included dichotomized functional outcome (mRS $\leq 2$ versus $m R S \geq 3$ ), successful revascularization, symptomatic reperfusion hemorrhage and mortality.

Results 108 patients met inclusion criteria, including 79 octogenarians (73\%) and 29 nonagenarians (27\%). Mean octogenarian age was 84.2 years (SD 2.8) versus 92 years (SD 2.3) in nonagenarians. Nonagenarians were more likely to be female $(86 \%$ versus $58 \%$; $<<0.01)$; there were no other differences between the groups in terms of demographics, medical comorbidities, pre-treatment clinical variables, or endovascular treatment characteristics. Median mRS at 90 days was 5 (IQR 3-6) in octogenarians and 6 (IQR 4-6) in nonagenarians $(p=0.09)$. Independent functional status $(m R S \leq 2)$ at 90 days was achieved in $13 \%$ of nonagenarians and in $20 \%$ of octogenarians $(p=0.54)$. Successful revascularization (TICI 2b-3) was achieved in $79 \%$ in both the octogenarian and nonagenarian cohorts $(p=1)$. Symptomatic reperfusion hemorrhage occurred in $21 \%$ of nonagenarians and in $6 \%$ of octogenarians $(p=0.03)$. The 90 -day mortality rate was $63 \%$ in nonagenarians versus $41 \%$ in octogenarians $(p=0.07)$.

Conclusions Nonagenarian patients undergoing EVT for AIS due to LVO are at significantly higher risk of symptomatic reperfusion hemorrhage compared with octogenarians, despite similar stroke- and treatment-related factors. While there was a strong trend towards higher mortality rates and worse longterm functional outcomes in nonagenarians, the difference was not statistically significant in this relatively small retrospective study. Additional prospective and randomized studies are necessary to evaluate the efficacy of EVT in elderly patients, including nonagenarians. 